\title{
Reflexiones sobre el uso de la Odds Ratio o la Razón de Prevalencias o Proporciones
}

\section{Considerations on the use of Odds Ratio versus Prevalence or Proportion Ratio}

\author{
Albert Espelt*,**,***, Marina Bosque-Prous*****, Marc Marí-Dell'Olmo**,**** \\ * Facultat de Ciències de la Salut de Manresa. Universitat de Vic Universitat Central de Catalunya (UVicUCC). Av. Universitària \\ 46, 08242, Manresa, Spain \\ ** Centro de Investigación Biomédica en Red de Epidemiología y Salud Pública (CIBERESP). C/ Monforte de Lemos 3 \\ Pabellón 11, 28029, Madrid, Spain \\ ***Departament de Psicobiologia i Metodologia en Ciències de la Salut. Universitat Autònoma de Barcelona (UAB). C/ de Ca \\ n’Altayó s/n. 08193 Bellaterra. Spain \\ ****Agència de Salut Pública de Catalunya, Barcelona, España; Departament de Ciències Experimentals i de la Salut, \\ Universitat Pompeu Fabra, Barcelona, España. \\ ***** Faculty of Health Sciences, Universitat Oberta de Catalunya, Barcelona, Spain.
}

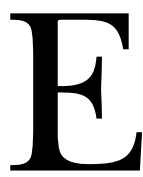
n 2017, publicamos un artículo explicando cómo estimar razones de prevalencia a partir de diferentes modelos de regresión (Espelt, Mari-Dell'Olmo, Penelo y Bosque-Prous, 2017). A menudo, en los trabajos de investigación en ciencias de la salud, trabajamos con estudios transversales o longitudinales. Estos estudios, tienen como variables de interés variables dicotómicas de enfermedad que representamos como proporciones, como las prevalencias o las incidencias acumuladas (por ejemplo, porcentaje de personas con consumo de riesgo de alcohol o porcentaje de nuevos casos de consumidores de riesgo de alcohol en un período determinado, respectivamente) (Hernandez-Avila, Garrido-Latorre y Lopez-Moreno, 2000; Moreno-Altamirano, López-Moreno y Corcho-Berdugo, 2000). En este sentido, cuando trabajamos con variables dependientes dicotómicas y con proporciones, lo primero que presentamos es la prevalencia o la incidencia acumulada de la enfermedad o comportamiento no saludable. Una vez localizada la prevalencia o incidencia acumulada, lo que nos interesará es ver si esta prevalencia o incidencia acumulada cambia en función de las distintas variables independientes o explicativas. Es por este motivo que después de una primera tabla de descripción de la muestra, solemos encontrar o querer presentar, una tabla donde se presentan proporciones según las distintas variables independientes o explicativas (con sus respectivas pruebas estadísticas). Hasta este momento, seguro que todas nos pondríamos de acuerdo. Sin embargo, muchas veces nos interesa mostrar las medidas de asociación entre estas variables dependientes dicotómicas y las variables independientes o explicativas de forma cruda y ajustada. En este punto, nos surge alguna duda, que ya intentamos responder en el artículo publicado en 2017 a partir de datos de un estudio europeo sobre alcohol (Espelt et al., 2017): ¿qué medida de asociación es mejor la Razón de Odds (OR) o la Razón de Prevalencias o Proporciones (RP)? ¿cuál deberíamos escoger? Si nos basamos en encontrar simplemente la asociación o las variables asociadas, la respuesta sería que no hay problema en utilizar una u otra de forma indistinta. Sin embargo, si vamos a interpretar la magnitud de esta asociación debemos tener claro que la OR y la RP no se interpretan de la misma forma. El problema es que la OR es difícil de comprender y habitualmente se interpreta como si fuera equivalente a una RP. Interpretar una OR como si fuera una RP se agrava si la prevalencia, proporción o incidencia acumulada de la enfermedad o comportamiento de salud es elevada (Szklo y Nieto, 2012), ya que la OR no se aproxima a una RP, sobreestimando la asociación. Por ejemplo, una OR de 2,65 puede ser perfectamente una RP de 2,08 (Espelt et al., 2017) y aquí empieza a surgir el problema. No es lo mismo decir que hay un $265 \%$ más de enfermedad en una categoría respecto a otra que $208 \%$. Si revisamos la literatura científica, encontraremos que la mayor parte de las publicaciones de estudios transversales

Recibido: Agosto 2019; Aceptado: Septiembre 2019.

Enviar correspondencia a:

Marina Bosque-Prous. Facultat de Ciències de la Salut. Universitat Oberta de Catalunya. Rambla del Poblenou, 156,08018 Barcelona E-mail: mbosquep@uoc.edu 
o longitudinales con variables dependientes dicotómicas se presentan las asociaciones con OR mientras que son pocas las que las presentan con RP (Espelt et al., 2017). Desde nuestro punto de vista, para dar coherencia al diseño, para que las tablas descriptivas de proporciones sean totalmente comparables con los datos crudos de asociaciones y para que no haya posibles errores en la interpretación de los datos de dichas asociaciones, lo más lógico sería utilizar la RP como medida de asociación, siempre que se pueda, para diseños con proporciones (transversales o longitudinales). Sobre todo, porque cuando se presentan OR en tablas hay mucha probabilidad que personas no especialistas interpreten de forma errónea la OR como RP y entiendan la OR como mayor probabilidad, mayor riesgo o más prevalencia cuando se debería interpretar como una comparación de probabilidades: la odds de que pase un evento respecto a la odds de que no pase ese mismo evento, es decir, la odds relativa. Pero también las personas epidemiólogas o investigadoras caen a veces en estos errores y encontramos artículos con vocabulario no adecuado para explicar dicha asociación (Barlés Arizón, Escario y Galbe Sánchez-Ventura, 2014; Díaz Geada, Busto Miramontes y Caamaño Isorna, 2018; Mori-Gamarra et al., 2018).

En este sentido, en el anterior artículo dimos los pasos para calcular razones de prevalencias con dos paquetes estadísticos STATA y R. En esta ocasión aprovechamos para facilitar también el procedimiento con el paquete estadístico SPSS, altamente utilizado en nuestro entorno, a partir de los menús. En la tabla 1, se puede ver como obtener las RP a partir del modelo de regresión de binomial utilizando SPSS. Es muy importante que la variable dependiente este codificada como 0 y 1 , siendo 1 la enfermedad o comportamiento de salud de interés.

Tabla 1. Explicación de los pasos para estimar la RP utilizando modelos de regresión log-binomial con SPSS

\begin{tabular}{l}
\hline Tool Bar (step by step) \\
\hline Menu tools $\rightarrow$ Analizar $\rightarrow$ Modelos Lineales Generalizados $\rightarrow$ Modelos \\
lineales generalizados $\rightarrow$ Tipo de modelo [Personalizado; Distribución: \\
Binomial; Función enlace: Logaritmo] $\rightarrow$ Respuesta [Variable \\
dependiente: auditc; Tipo de variable dependiente: Binario; Categoría \\
de referencia: Primera (menor valor)] $\rightarrow$ Predictores [Factores: educ, \\
sex; Covariables: age] $\rightarrow$ Modelo [Modelo: educ, sex, age] $\rightarrow$ Estimación \\
[iteraciones máximas: 100000$] \rightarrow$ Estadísticos [Incluir estimaciones de \\
los parámetros exponenciales]
\end{tabular}

En muchos artículos cuando analizamos la asociación entre una variable dependiente y una o varias variables independientes o explicativas intentamos controlar por posibles variables que podrían ser un factor de confusión (Babyak, 2009). Una de las técnicas estadísticas empleadas para controlar por otras variables es el uso de modelos de regresión. En los distintos estudios, a partir de modelos de regresión, encontramos asociaciones crudas y asociaciones ajustadas por alguna de estas variables de confusión. En este sentido, por ejemplo, sabemos que vivir en un entorno urbano es protector del consumo de alcohol respecto a vivir en un entorno rural $[\mathrm{RP}=0,86(\mathrm{IC} 95 \%=0,78-0,95)]$. Sin embargo, este efecto protector desaparece $[\mathrm{RP}=0,91$ (IC95\% $=0,80$ $1,05)$ ] cuando se tienen en cuenta distintas variables individuales y aspectos como los centros deportivos, la tasa de desempleo, el número de pubs, y otras variables de accesibilidad (Obradors-Rial, Ariza, Continente y Muntaner, 2019).

En el artículo citado es fácil ver, a partir de la tabla de prevalencias, que la prevalencia de consumo de riesgo de alcohol en el entorno urbano $(51,1 \%)$ es menor que la del entorno rural (59,3\%) (Obradors-Rial et al., 2019). Una simple división de la prevalencia de consumo de riesgo de alcohol en el entorno urbano entre la prevalencia de consumo de riesgo de alcohol del entorno rural nos revela la asociación dada por el modelo de regresión $(\mathrm{RP}=0,86)$. Ahora bien, hemos dicho que la asociación ajustada entre consumo de riesgo de alcohol y entorno urbano/rural es de 0,91. Podemos saber, ¿cuál es la prevalencia ajustada de consumo de alcohol en el entorno urbano y en el entorno rural que nos da esta asociación? Con el programa estadístico STATA y R, podemos estimar la prevalencia ajustada para cada una de las categorías de la variable independiente de interés (Muller y MacLehose, 2014). Si volvemos al artículo publicado en 2017 (Espelt et al., 2017), en la tabla 2 , podemos ver que la prevalencia de bebedores de riesgo en los hombres de Estonia era de 16,75\% mientras que en las mujeres de $4,24 \%$, lo que implica una $\mathrm{RP}=3,95$. La Razón de Prevalencia ajustada por edad y nivel de estudios de Estonia era de 3,87. Ahora bien, aplicando el modelo de regresión y calculando sus marginales (tabla 2), podemos saber que la prevalencia de bebedores de riesgo de los hombres ajustada por edad y nivel de estudios era de 16,48\% y el de las mujeres de 4,26\%. Si aplicamos la división de las prevalencias de bebedores de riesgo en hombres y mujeres obtenemos la misma RP ajustada que la del modelo.

Tabla 2. Sintaxis para obtener las prevalencias crudas y ajustadas con STATA y $R$.

\begin{tabular}{l}
\hline Sintaxis modelo ajustado \\
\hline STATA \\
\hline glm auditc i.sex i.educ age, family(binomial 1) link(log) eform \\
margins i.sex i.educ \\
\hline $\mathbf{R}$ \\
\hline install.packages(pkgs =c(“Epi”, “foreign")) \\
library(Epi) \\
library(foreign) \\
model<-glm(auditc sex + educ + age, data=data, \\
family=binomial(link=log)) \\
summary(model) \\
round(ci.lin(model, Exp=T),2) \\
library(prediction) \\
pred ८- prediction(model, at = list(sex=c(“Women”,"Men”)), type = \\
"response”)
\end{tabular}


En conclusión, desde nuestro punto de vista, en estudios en los que la variable independiente o de interés es una proporción, creemos que es más útil utilizar como medida de asociación las razones de prevalencia o proporciones ya que supone una mayor coherencia y evita errores de interpretación.

\section{Conflicto de intereses}

Las personas autoras declaran que no existe ningún conflicto de interés. Albert Espelt es editor asociado de la revista Adicciones. Sin embargo, no ha tenido ningún papel durante el proceso editorial.

\section{Bibliografía}

Babyak, M. A. (2009). Understanding confounding and mediation. Evidence-based Mental Health, 12, 68-71. doi:10.1136/ebmh.12.3.68.

Barlés Arizón, M., Escario, J. y Galbe Sánchez-Ventura, J. (2014). Predictors of driving under the influence of alcohol among Spanish adolescents. Adicciones, 26, 96-105.

Díaz Geada, A., Busto Miramontes, A. y Caamaño Isorna, F. (2018). Alcohol, tobacco and cannabis consumption in adolescents from a multicultural population (Burela, Lugo). Adicciones, 30, 264-270. doi:10.20882/adicciones.915.

Espelt, A., Mari-Dell'Olmo, M., Penelo, E. y Bosque-Prous, M. (2017). Applied Prevalence Ratio estimation with different Regression models: An example from a cross-national study on substance use research. Adicciones, 29, 105-112. doi:10.20882/adicciones.823.

Hernandez-Avila, M., Garrido-Latorre, F. y Lopez-Moreno, S. (2000). Epidemiologic study design. Salud Pública de México, 42, 144-154.

Moreno-Altamirano, A., López-Moreno, S. y Corcho-Berdugo, A. (2000). Principales medidas en epidemiología. Salud Pública de México, 42, 337-348.

Mori-Gamarra, F., Moure-Rodríguez, L., Sureda, X., Carbia, C., Royé, D., Montes-Martínez, A., ... Caamaño-Isorna, F. (2018). Alcohol outlet density and alcohol consumption in Galician youth. Gaceta Sanitaria, pii: S02139111(18)30238-3. doi:10.1016/j.gaceta.2018.09.005.

Muller, C. J. y MacLehose, R. F. (2014). Estimating predicted probabilities from logistic regression: Different methods correspond to different target populations. International Journal of Epidemiology, 43, 962-970. doi:10.1093/ ije/dyu029.

Obradors-Rial, N., Ariza, C., Continente, X. y Muntaner, C. (2019). School and town factors associated with risky alcohol consumption among Catalan adolescents. Alcohol, pii: S0741-8329(17)30987-4. doi:10.1016/j.alcohol.2019.04.005.
Szklo, M. y Nieto, J. (2012). Epidemiology: Beyond the Basics (3 edition). Burlington, Mass: Jones \& Bartlett Learning. 